\title{
Microstructure Evolution and Deformation Behavior of AZ31 Magnesium Alloy During Alternate Forward Extrusion
}

\author{
Feng $\mathrm{Li}^{1} \cdot$ Yang $\mathrm{Liu}^{1} \cdot$ Xubo $\mathrm{Li}^{1}$
}

Received: 1 June 2017/Revised: 4 August 2017/Published online: 20 September 2017

(C) The Chinese Society for Metals and Springer-Verlag GmbH Germany 2017

\begin{abstract}
The paper presents a new extrusion method, alternate forward extrusion, in which the punch was replaced with double-split structures so as to achieve the grain refinement for material near the interface of double-split structures. The results showed that the unique loading mode made metal flow sequence and behavior significantly changed during alternate forward extrusion. The additional shear deformation produced by the double-split punch structures resulted in a refining effect on the microstructure of the blank, which was then further refined during flow through the die orifice owing to shear deformation. Compared with the conventional extrusion, the recrystallization process in the alternate forward extrusion process produced grains that were smaller and more homogeneous in size. The recrystallization process was more abundant, and the dislocation density was significantly increased. It can be concluded that the alternate forward extrusion process could achieve fine-grained strengthening, which provided technical support and scientific guidance for the engineering application of magnesium alloy extrusion forming technology.
\end{abstract}

\section{KEY WORDS: Magnesium alloy; Alternate forward extrusion; Microstructure evolution; Deformation} behavior

\section{Introduction}

The strong three-dimensional compressive stress state during extrusion can refine metal grains owing to shear deformation of the die orifice; therefore, the extrusion process is especially suitable for forming low-plasticity magnesium alloy $[1,2]$. The microstructure and properties of magnesium alloy were studied by scholars from the aspects of forming temperature, extrusion ratio and alloying [3, 4]. Kim et al. [5] investigated the microstructure and mechanical property of an AZ31 Mg alloy subjected to

Available online at http://link.springer.com/journal/40195

Feng Li

fli@hrbust.edu.cn; hitlif@126.com

1 School of Materials Science and Engineering, Harbin University of Science and Technology, Harbin 150040, China low-temperature indirect extrusion with artificial cooling. The grain size was considerably reduced from 5.5 to $1.8 \mu \mathrm{m}$ increasing yield strength to as high as $50 \mathrm{MPa}$, exhibiting elongation of $200-320 \%$ at strain rates in the range of $3.3 \times 10^{-5}-1.0 \times 10^{-4} \mathrm{~s}^{-1}$ at $423 \mathrm{~K}$. Chandrasekaran et al. [6] studied the extrudability of three different magnesium alloys (namely AZ31, AZ61 and ZK60 alloys) at various temperature conditions. Although the forward extrusion of magnesium alloys at temperatures of $423 \mathrm{~K}$ was possible, there was difficulty in removing the specimens from the die cavity. Extrusion of AZ31 magnesium alloys below temperatures of $423 \mathrm{~K}$ was possible with application of suitable counter pressure. Shanzad et al. [7] performed an extrusion test of the DC-cast alloy AZ80 in the temperature range of 448-623 $\mathrm{K}$, and revealed that the extrusion temperature influences the modes of plastic deformation and the dynamic recrystallization (DRX) response. At lower temperatures, twinning was more active, DRX grains nucleated at the twin boundaries and a 
fine grain size was achieved. At higher temperatures, new grains nucleated at the original grain boundaries and a relatively coarse grain size were achieved. Borkar et al. [8] researched the texture distribution of M1-Sr alloys extrudates after extrusion at different speeds and discovered that the strong basal fiber texture in extruded M1 weakened with $\mathrm{Sr}$ addition. The weakened of texture was more pronounced at higher speed extrusion than lower speed extrusion and obviously improved the mechanical properties. Murai et al. [9] proposed that the billet homogenization and extrusion parameters affect the microstructure and mechanical properties of AZ31B magnesium alloy. Borkar et al. [10] reported the texture strength of $\mathrm{Mg}-1 \% \mathrm{Mn}$ (M1) alloy after extrusion at different temperatures. M1 alloy exhibited weaker texture after extrusion at $573 \mathrm{~K}$, but developed strong basal texture at 623 and $673 \mathrm{~K}$. To improve the existing magnesium alloy extrusion process and the loading mode, some new methods have been developed, such as forward extrusion through a rotating container [11], continuous variable cross section direct extrusion [12] and differential speed extrusion [13].

In conclusion, the extrusion process has been studied and widely used in the field of high-performance magnesium alloy forming. In particular, severe plastic deformation (SPD) is promising because it allows multiple loadings, which can obtain high-performance products because of the larger cumulative strain $[14,15]$. This kind of technology still needs to be repeated several times for the blank and it is limited to laboratory research stage [16]. The focus of current research in this direction is to improve the grain refinement ability using SPD in a single pass.

\section{Experimental Procedure}

\subsection{Process Principle}

Inspired by accumulative back extrusion (ABE), this paper combines the loading mode of downward alternately with the conventional extrusion (CE) [17, 18]. Then we put forward the alternate forward extrusion (AFE) method and draw the schematic as shown in Fig. 1.

The unique characteristic of the AFE was that the traditional loading mode of forward extrusion was changed into the interactive loading mode. The actual loading function area of single split punch significantly decreased in the loading process; therefore, the forming load was obviously reduced. The metal flow behavior and order in the mold cavity were changed when the two punches was alternately loaded. In addition severe shear deformation at the interface of different punch end deeply refined the billet internal organization, which had important influence on the evolution of microstructure. A full load pass included two steps. First, the right punch fixed and the left punch moved downward for $\mathrm{h}$. Then, the left punch fixed and the right punch moved downward to the same position subsequently. In this way, the two punches alternately applied load downward and the billet are processed for three passes.

\subsection{Materials and Methods}

Commercially available casting feedstock of AZ31 alloys rod was used for the sample material in the experiment, and the chemical composition is shown in Table 1.

Cast rod was lathed to ingots with dimensions of $\Phi 40 \mathrm{~mm} \times 50 \mathrm{~mm}$. Then the ingots were homogenized treatment at $693 \mathrm{~K}$ for $12 \mathrm{~h}$ followed by cooling to room temperature before extruded. In order to reduce friction, water-based graphite was used as a lubricant. Single pass press length $\mathrm{h}$ set to $10 \mathrm{~mm}$. The billet was hot extruded at $573 \mathrm{~K}$ using AFE method under an extrusion rate of $1 \mathrm{~mm} /$ $\mathrm{s}$ and an extrusion ratio 8.16. The AFE process was terminated when the ingots had been extruded to about $240 \mathrm{~mm}$ from the die and the extruded AZ31 alloy embedded in the oriented die was removed from the die and then quenched in water in order to investigate the microstructure evolution during AFE.

In order to avoid the periodic "S shape" defects of the AFE extrudates, the oriented die was designed and adopted in this study. The linearity of the interrupted extrudate is better as shown in Fig. 2. The extrudates was sectioned along a plane containing the extrusion axis. Five characteristic locations, labeled A, B, C, D, and E, respectively, were selected at the sectioned sample for further analysis of microstructure evolution from start to end during AFE.

After the AFE tests, optical microscopy (OM) and electron backscattered diffraction (EBSD) were conducted for microstructure analyses at five characteristic locations. The extrusion direction (ED), normal direction (ND) and transverse direction (TD) were shown in Fig. 2. The observation planes were along vertical extrusion direction plane (ND and TD plane) and parallel to the extrusion direction plane (ED and TD plane), respectively. The specimens for OM were ground with 1200 grit $\mathrm{SiC}$ paper, and then mechanically polished using diamond paste, and etched in a solution of $1 \mathrm{ml}$ acetic acid, $1 \mathrm{ml}$ nitric acid, $1 \mathrm{~g}$ oxalic acid and $150 \mathrm{ml}$ water. The specimens for EBSD were ground with 2000 grit $\mathrm{SiC}$ paper, followed by mechanically polishing by diamond paste, then prepared by electro-polishing in an electrolyte solution which consisted of phosphoric acid and ethyl alcohol (volume ratio 3:5) at $0.5 \mathrm{~A}$ for $2 \mathrm{~min}$ and then at $0.2 \mathrm{~A}$ for $3 \mathrm{~min}$ for the final stage. The EBSD tests were carried out by a $200{ }^{\circ} \mathrm{F}$ Quanta field emission environmental scanning electron microscope, and the experimental data were analyzed by OIM Analysis TSL 6 software. The step size of EBSD 


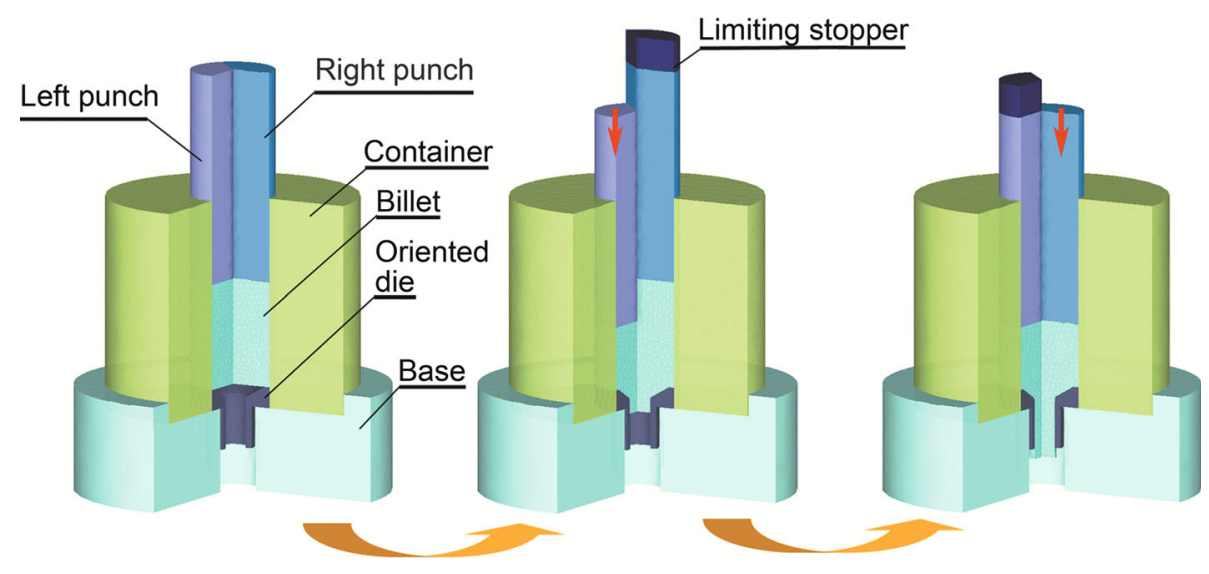

Fig. 1 Schematic of AFE

Table 1 AZ31 magnesium alloy composition

\begin{tabular}{llllllll}
\hline $\mathrm{Al}$ & $\mathrm{Zn}$ & $\mathrm{Mn}$ & $\mathrm{Fe}$ & $\mathrm{Si}$ & $\mathrm{Cu}$ & $\mathrm{Ni}$ & $\mathrm{Mg}$ \\
\hline 3.20 & 0.86 & 0.36 & 0.0018 & 0.021 & 0.0022 & 0.00056 & Bal.
\end{tabular}

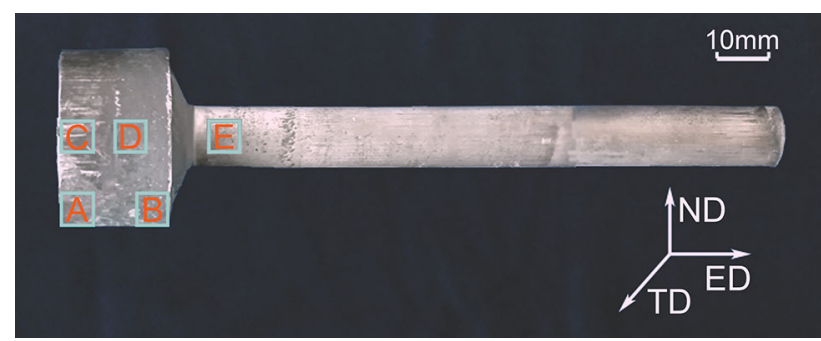

Fig. 2 Interrupted extrudate with five characteristic locations labeled $\mathrm{A}, \mathrm{B}, \mathrm{C}, \mathrm{D}$, and $\mathrm{E}$

measurements was $1.3 \mu \mathrm{m}$. The microstructures of vertical extrusion direction plane (ND and TD plane) were observed by JEOL JEM-2100 transmission electron microscopy (TEM).

\section{Results and Discussion}

\subsection{Shear Strain}

Shear deformation is an important factor for grain refinement and in bulk forming process [19]. There only exists large shear deformation around the die orifice during CE. In order to promote deformation, it is necessary to increase the extrusion ratio [20], which will significantly increase the extrusion load, damage the die life and generate some defects in extrudates. Due to the unique loading mode, the AFE process can provide the additional shear deformation at the interface of the double-split punches. The DEFORM software was used to study the distribution of shear strain in the present research and the process conditions strictly set in accordance with the experimental conditions. The shear strain distribution of AFE and CE is shown in Fig. 3 for punch down strokes of 10, 20 and $30 \mathrm{~mm}$. Figure $3 \mathrm{a}$ shows equidistant point position in which was used to measure the shear strain values.

Although adopting different extrusion processes, the variation of the shear strain at the point location during the two kinds of extrusion processes is more regular in Fig. 3. The shear strain reached the minimum and maximum at the end of the punch and die orifice, which increased with the increase in the distance from the punch end in CE. Compared with the CE, the additional shear deformation which can be provided by AFE was obvious. With the increase in the distance from the punch end, the shear strain firstly decreased, and then increased. Shear strain values were listed from a high level to a low level: under the interface of the double-split punches, die orifice and the billet center of interrupted extrudate. The results showed that the shear strain value at the die orifice of the AFE is slightly lower than that of $\mathrm{CE}$ at the extrusion initial stage. The shear strain value at the die orifice was obviously higher than that of the $\mathrm{CE}$ and the growth was larger with the increase in punch down stroke. The shear strain value on the other location of the axis was also significantly higher than CE.

The shear deformation during extrusion process provided sufficient driving force for recrystallization nucleation [19], which was one of the effective ways to realize the grain deeply refinement. The additional shear deformation provided by AFE had an important influence on the microstructure evolution.

\subsection{Metal Flow}

Due to the double-split punches alternately applied load downward, the metal flow in each location of the billet had a sequential order in the extrusion process. The billet was 

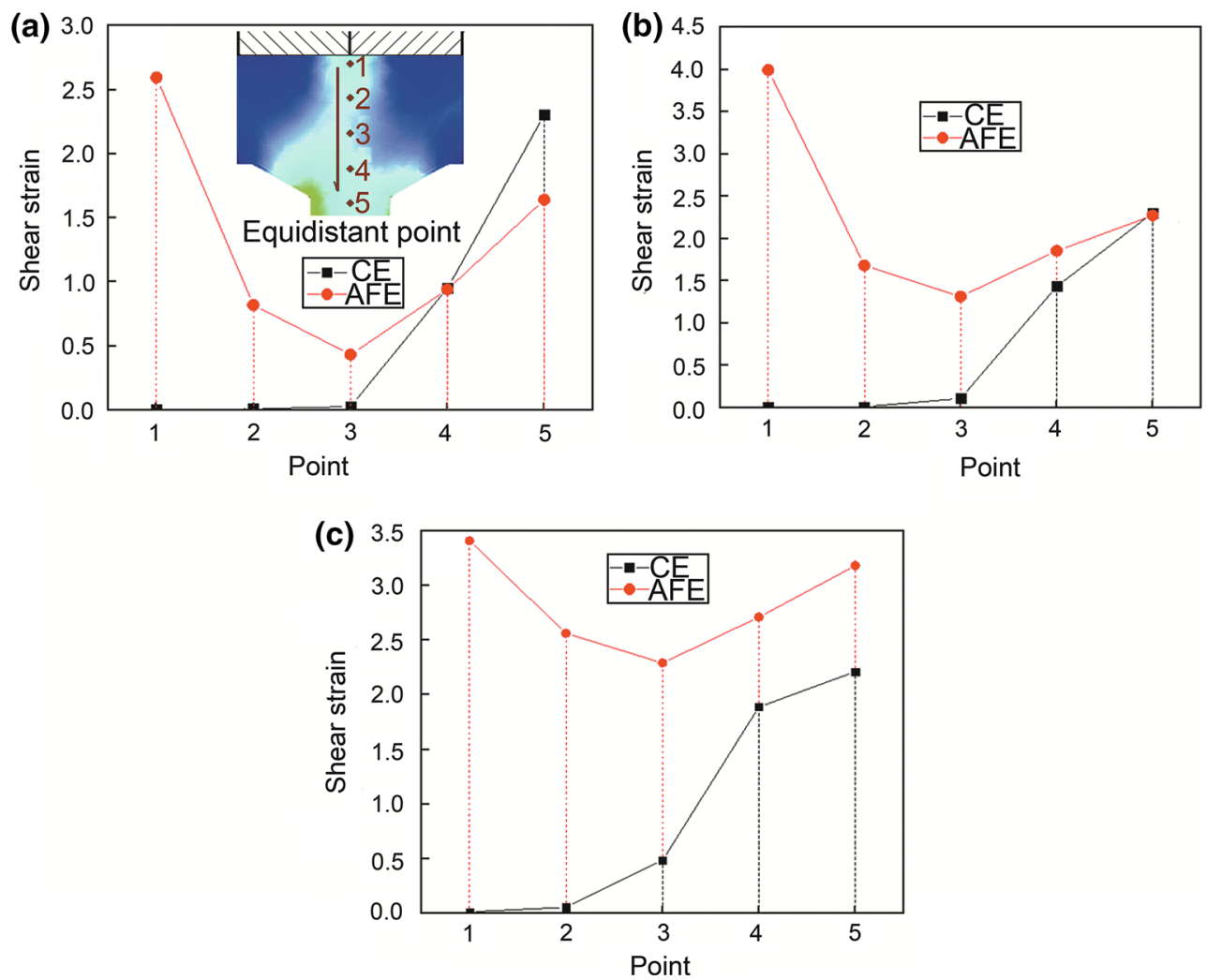

Fig. 3 Shear strain distribution under different punch down stroke a with $10 \mathrm{~cm}$ punch down stroke $\mathbf{b}$ with $20 \mathrm{~cm}$ punch down stroke $\mathbf{c}$ with $30 \mathrm{~cm}$ punch down stroke

extruded along the axial direction; and simultaneously had a flow tendency along the radial direction with the punch down loading. Because the metal flow behavior in the AFE process was similar, the flow behavior in the single pass was analyzed. The velocity field distribution of the billet in the container during the single pass showed in Fig. 4, with 20-30 $\mathrm{mm}$ double punches down stroke.

Figure $4 \mathrm{a}$ is the velocity field when the left punch applied load downward and the right punch fixed at the same time with the $20 \mathrm{~mm}$ double punches down stroke. It was not only the final state of the last loading pass but also the beginning of the next loading pass. When the left punch continue to apply load downward a single pass (that was $10 \mathrm{~mm}$ ), the velocity field showed in Fig. 4b. Due to the left punch was non-coaxial with the extrusion axis, most of the metal billet under it produced lateral flow toward the die orifice direction and was directly extrusion forming. At this time, the right punch was fixed and the metal billet was subjected to the adjacent radial thrust, resulting in the separated flow behavior. On the one hand, the metal located in the bottom portion of oriented die produced deformation flow toward the die orifice direction; on the other hand, the material near the end of the right punch was hindered by the bottom metal and the side wall of the container. So the metal close to the right punch end formed counterclockwise vortex flow and gradually filled the cavity formed by the two punch interface.

The right punch began to apply load downward when the loading single pass of left punch was completed and fixed. Figure $4 \mathrm{c}$ shows most of the metal billet under right punch produced lateral flow toward the die orifice direction. The metal close to the left punch end formed clockwise vortex flow and the same tendency that the metal located in the bottom portion of oriented die produced deformation flow toward the die orifice direction. Therefore, there was a flow interface between the separated flows. In Fig. 4d, when the double-split punches had the same down stroke, the entire load cycle finished.

The above analysis showed that during AFE process, the material flow near the end of the fixed punch certainly produced vortex flow behavior. The peculiar distribution of the velocity field had a certain influence on stress state and microstructure of billet. At the same time, it can be seen that the variation law of vortex size in one pass load cycle was: produced, increased, decreased, disappeared and reproduced. This was consistent with the change of the range of the corner region between the double-split punches in the AFE. 
(a)

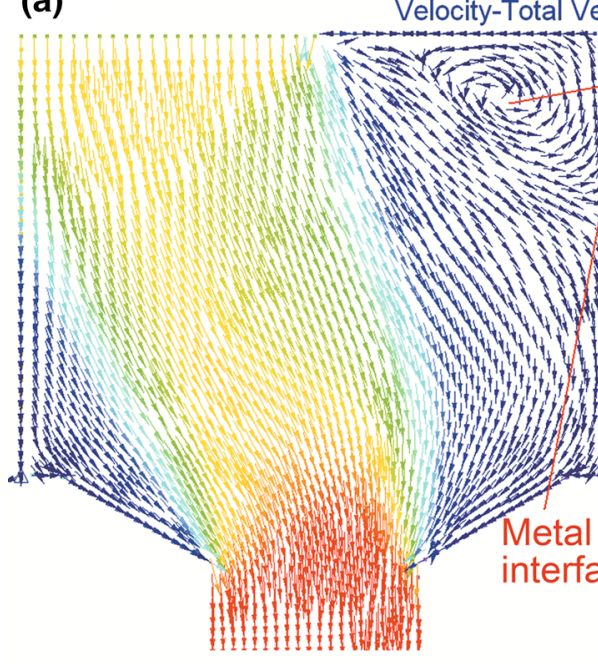

(d)

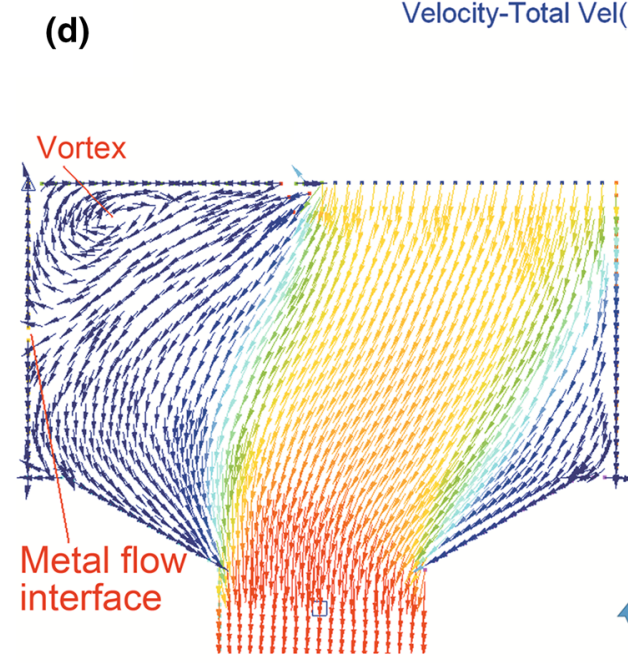

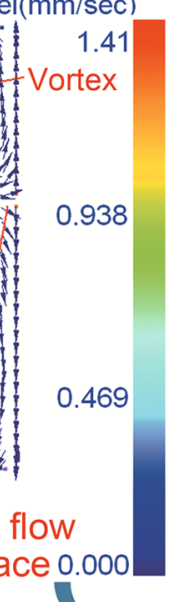

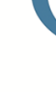

1.42

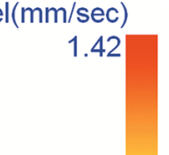

(c)

(c) (b)
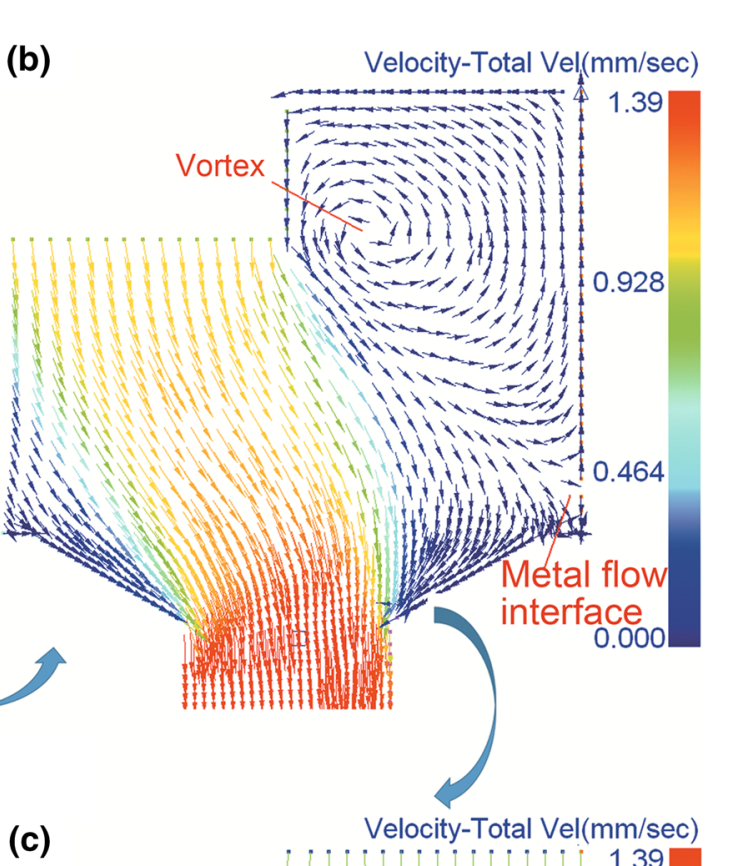

Fig. 4 Change of velocity field with different punch down stroke: a the left punch applied load downward with 20 cm punch down stroke when the right punch fixed, b the left punch applied load downward with $30 \mathrm{~cm}$ punch down stroke when the right punch fixed, c the right punch applied load downward with $20 \mathrm{~cm}$ punch down stroke when the left punch fixed, d the right punch applied load downward with $30 \mathrm{~cm}$ punch down stroke when the left punch fixed

\subsection{Grain Morphology}

The metal flow behavior and shear deformation revealed different characteristic locations during the AFE process at $573 \mathrm{~K}$. The grain morphology at five different characteristic locations showed in Fig. 5 with $30 \mathrm{~mm}$ double punches down stroke.

Firstly, a comparative study was made on the grain morphology of two locations (A, B) on the side wall of the container. In Fig. 5a, there were a lot of recrystallized grains around the location $\mathrm{A}$ and the average grain size was petty small. According to the previous analysis, the metal flow behavior and the complex stress state near the wall caused the above phenomenon. On the one hand, the material moved toward the die orifice direction, the interfacial friction force at the location A was opposite to the ED when the split punch applied load downward. On the other hand, due to the material produced vortex flow along the wall from bottom to top, the interface friction force at the location A was consistent with the ED when the split punch was fixed. The influence of the cyclic friction force on the grain refinement still needed to be further confirmed. In comparison, the material always moved toward the die orifice direction and the interface friction at the location $\mathrm{B}$ was opposite to the ED. Figure $5 \mathrm{~b}$ is the unhomogeneous microstructure at the location $\mathrm{B}$ which contained some recrystallized grains and some elongated grains. 


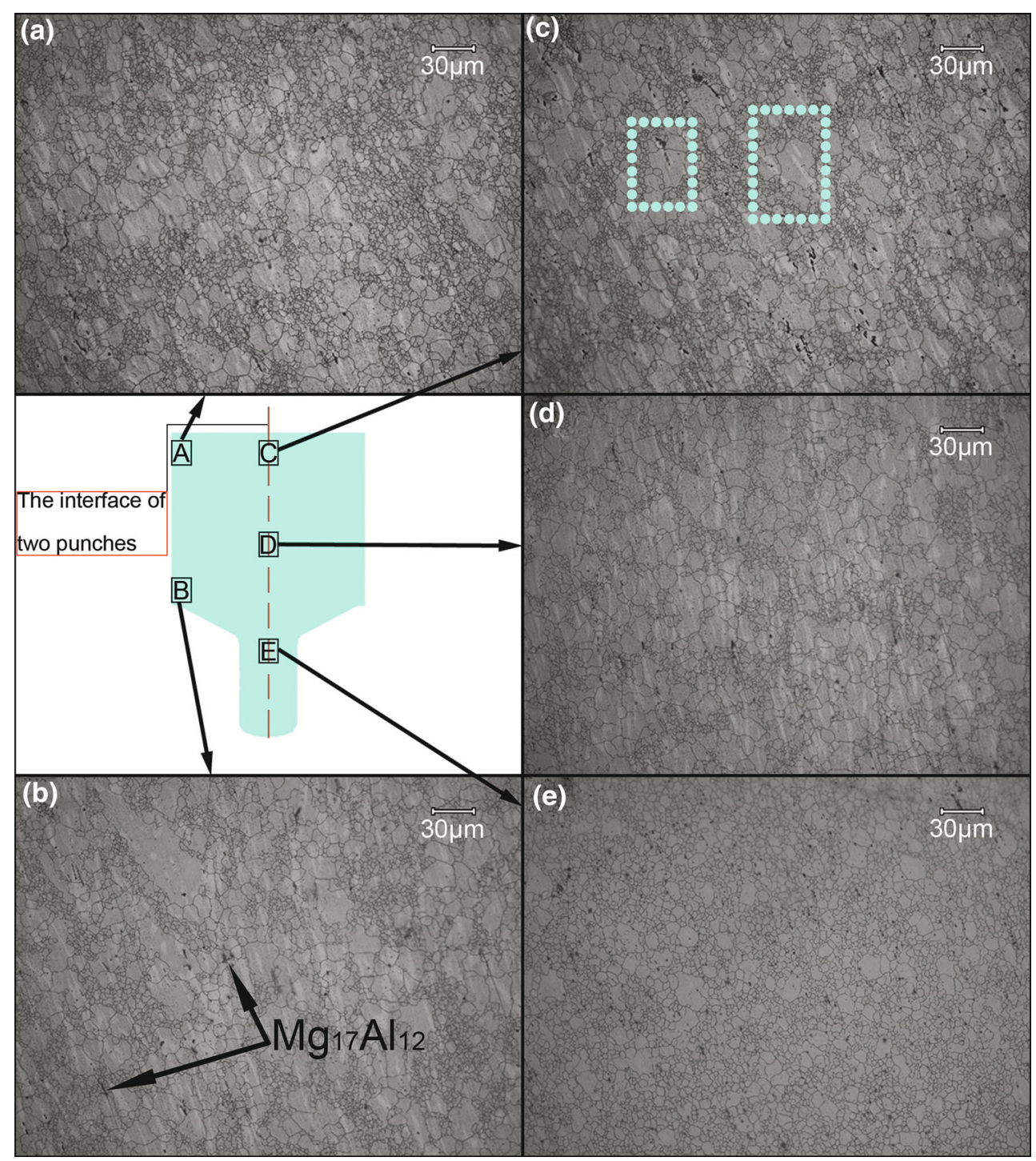

Fig. 5 Microstructures of the characteristic locations: a location A, b location B, $\mathbf{c}$ location $\mathrm{C}$, d location D, e location $\mathrm{E}$

Secondly, the change of the microstructure at the axis was studied. At location C (Fig. 5c), both coarse and recrystallized grains were present, which formed a typical "bimodal microstructure" [21]. This kind of microstructure was well studied by many scholars. Fine grains were distributed around the coarse grains boundaries, which formed a typical necklace structure marked by the rectangle. The grain microstructure at location $\mathrm{C}$ was significantly refined because of continuous, severe shear deformation at the interface of the double-split punches [19]. At location D, as shown in Fig. 5d, there were a few fine grains and a large number of coarse grains. The average grain size was relatively large because of the small shear deformation experienced at this location. From the above analysis, we can see that the shear deformation of the extrusion die orifice was larger. At location E, as shown in Fig. 3e, the microstructure morphology was typical DRX [15]. The grains at this location were round and homogeneous in size. The average grain size was $4.73 \mu \mathrm{m}$, reflecting a remarkable refining effect.

\subsection{Grain Orientation}

Figure 6 is the grain orientation maps of different locations when the double-split punch down stroke was $30 \mathrm{~mm}$. Different colors represent different crystallographic orientation. To improve the accuracy of the results, the grains less than $4 \mu \mathrm{m}$ are cleared and the grain tolerance angle is $5^{\circ}$.

The flow behavior of the metal made the grains orientation difference enlarging when the one punch applied load downward and the other punch fixed at the same time. In Fig. 6b, the crystallographic orientation of the grains at locations B obviously turned toward the lower left, so a 


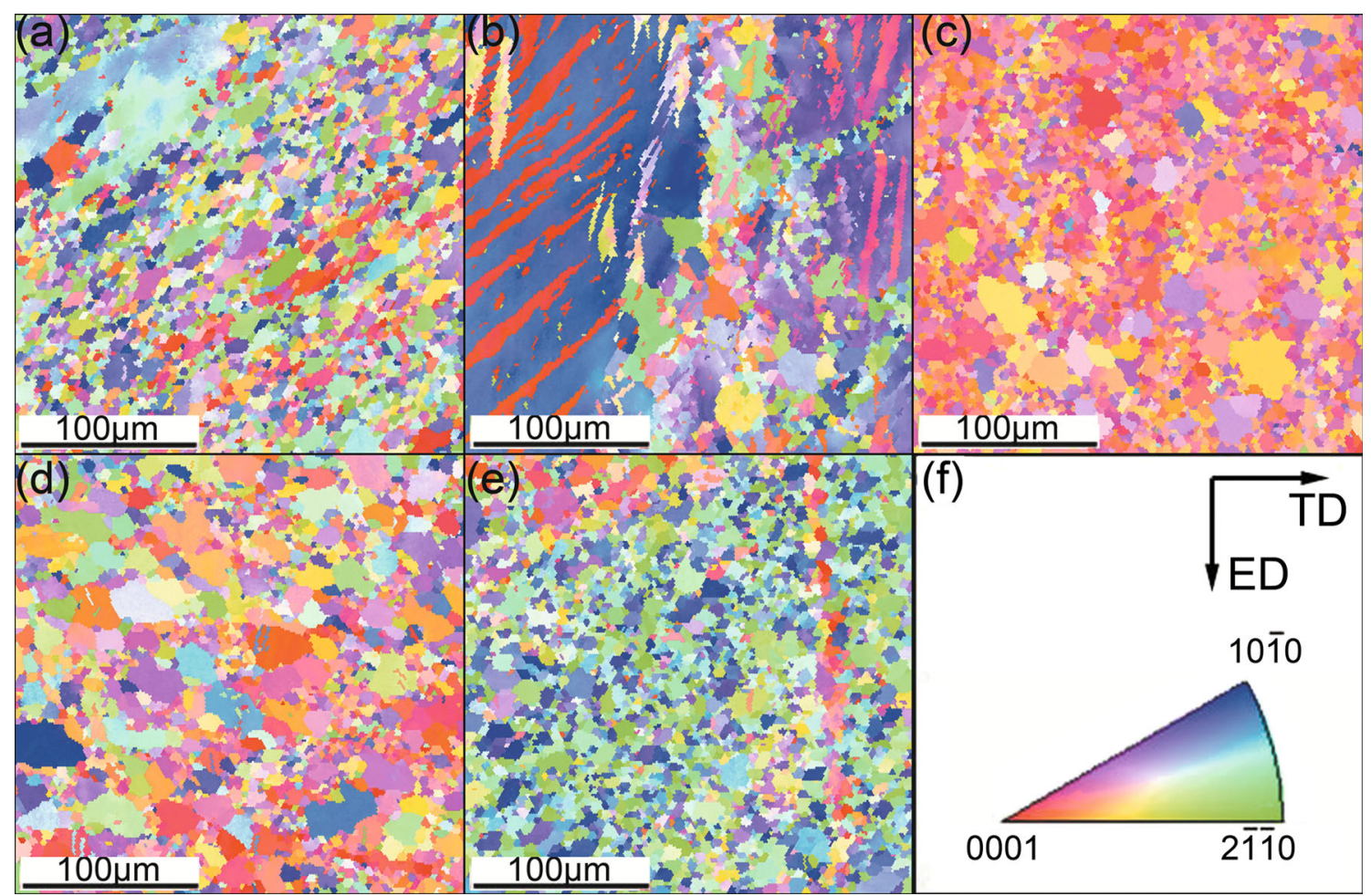

Fig. 6 Crystallographic orientation of the characteristic locations: a location A, b location B, $\mathbf{c}$ location C, d location D, e location E, $\mathbf{f}$ EBSD inverse pole figures (IPF)

large area of texture was formed [7, 12]. Due to the obstacle of the bottom metal, the material produced vortex flow along the container wall from bottom to top. At location A, as shown in Fig. 6a, the grains crystallographic orientation was rather disorder, resulting in weakening of the texture formed in locations B. These results were consistent with the simulation results of the metal flow behavior.

The crystallographic orientation at the three locations along the axis was analyzed in the same way. In Fig. 6c, although the location $\mathrm{C}$ at the interface of the double-split punches, the crystallographic orientation was almost parallel to the base surface because the double-split punches down stroke was identical. Only a small number of the grains had a tiny rotation, so formed a large range of the basal plane texture. As far away from the end of the punch, the grains at the location D had a significant rotation and most of the grains were not parallel to the base plane (Fig. 6d). At location E, as shown in Fig. 6e, most of the grains were obviously rotated except that the grains near the right side were still parallel to the basal plane. It was beneficial to weaken the basal plane texture of extrudate $[12,15]$.

In summary, the grain orientation of each location changed owing to the continuous loading of the doublesplit punches during the AFE process, which was helpful to weaken the basal plane texture.

\subsection{Grain Boundary Angle Analysis}

The research of misorientation angle distribution is helpful for the evaluation of DRX in microstructure. Recrystallized grains often have high grain boundaries [22], which have been confirmed by many scholars. The misorientation angle distribution images of five characteristic locations are shown in Fig. 7a-e. Based on the misorientation angle of each location, the average misorientation angle of the corresponding region could be obtained (Fig. 7f).

The average misorientation angle at the location $\mathrm{D}$ was the smallest (Fig. 7f), which indicated that the proportion of DRX was least. The average misorientation angle at the location E was the largest, and it could be seen that the proportion of DRX was biggest. The morphology and size of the grains were different in the container owing to the difference of the location and the extent of suffering local shear deformation. All the materials eventually flowed toward the die orifice (the location E), in which the shear deformation was largest. The DRX process was more complete due to a certain "integration" treatment during extrusion [21].

\subsection{Mechanism Analysis}

Figure 8 is TEM images at different locations when the double-split punch down stroke was $30 \mathrm{~mm}$. Figure $8 \mathrm{a}$ 

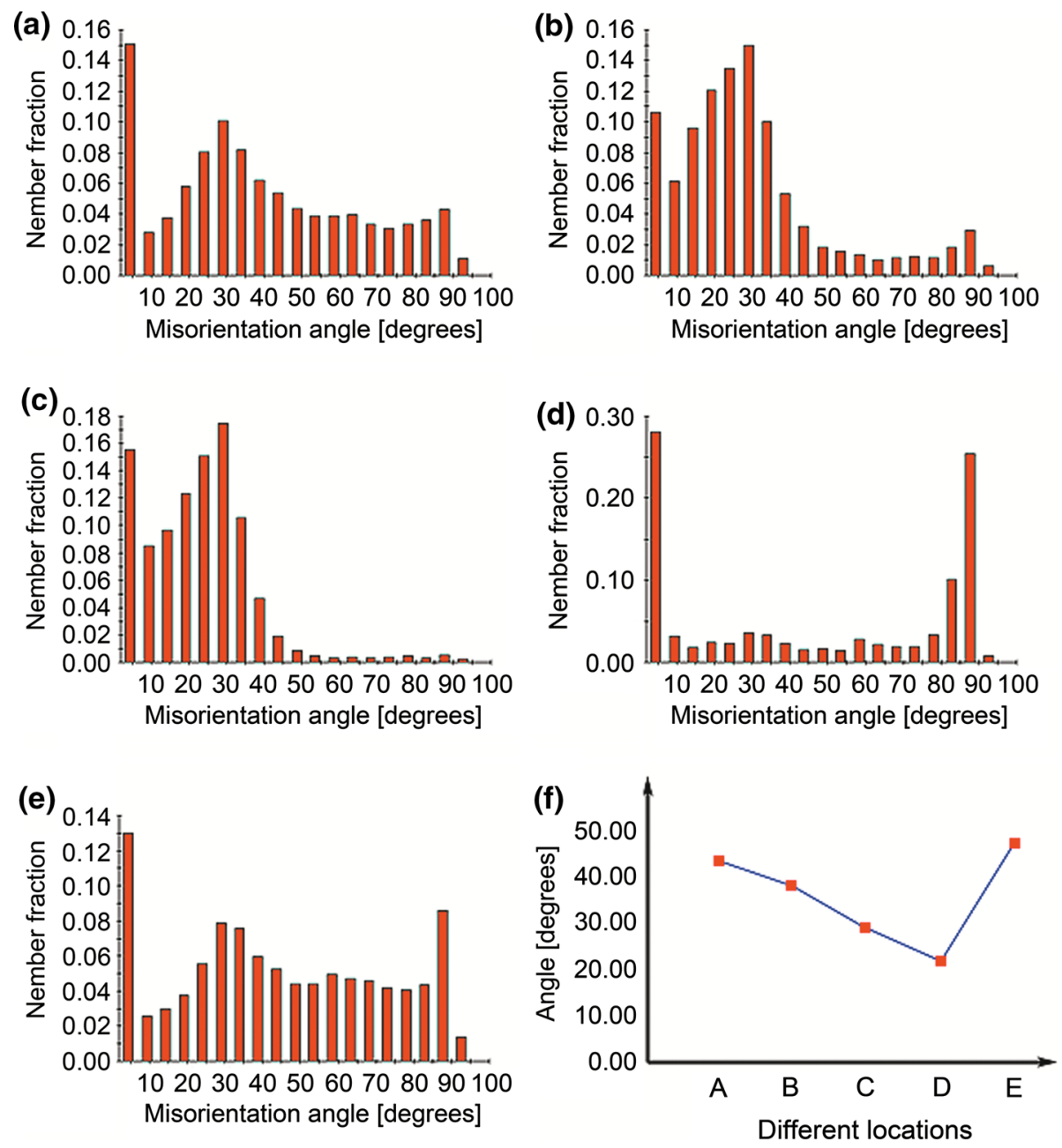

Fig. 7 Misorientation angle distribution of the characteristic locations at location A (a), location B (b), location C (c), location D (d), location E (e) and the average misorientation angle variation of the corresponding region (f)

shows the fine DRX grains observed at location A. At the same time, the large grains could be divided into subgrains which might be the source of a large number of DRX grains in Fig. 8b. According to the previous analysis, the friction force between the "vortex flow" and container also could form DRX.

At location B, as shown in Fig. 8c, a large number of elongated grains were observed. The twins were interspersed between elongated grains. This might be owing to the metal flow formation of "vortex flow," which made material difficult to deform further. In order to make the deformation continue, the twins were formed to release the internal stress. In addition, there was a large difference in the grain orientation, which could be seen from the contrast change of the adjacent grains in Fig. 8d.

At location C, as shown in Fig. 8e, the DRX [15] had a high degree of roundness and small grain size. The strong dislocations were entangled with each other to form a dislocation net in Fig. 8f. The main reason for these phenomena was the strong shear deformation $[17,19]$ in the billet near the double-split punches.

The location D located in the middle of the billet and not affected by the large shear deformation. Figure $8 \mathrm{~g}$ and $\mathrm{h}$ showed a triple grain boundary and a lower dislocation density.

Location $\mathrm{E}$ had strong dislocation and densely distributed DRX in Fig. 8i [12, 21]. The microstructure was obviously refined because of the severe shear deformation in this area. The subgrains were separated from the small grains which formed a new grain boundary owing to the gradual absorption of dislocations (Fig. 8j). 

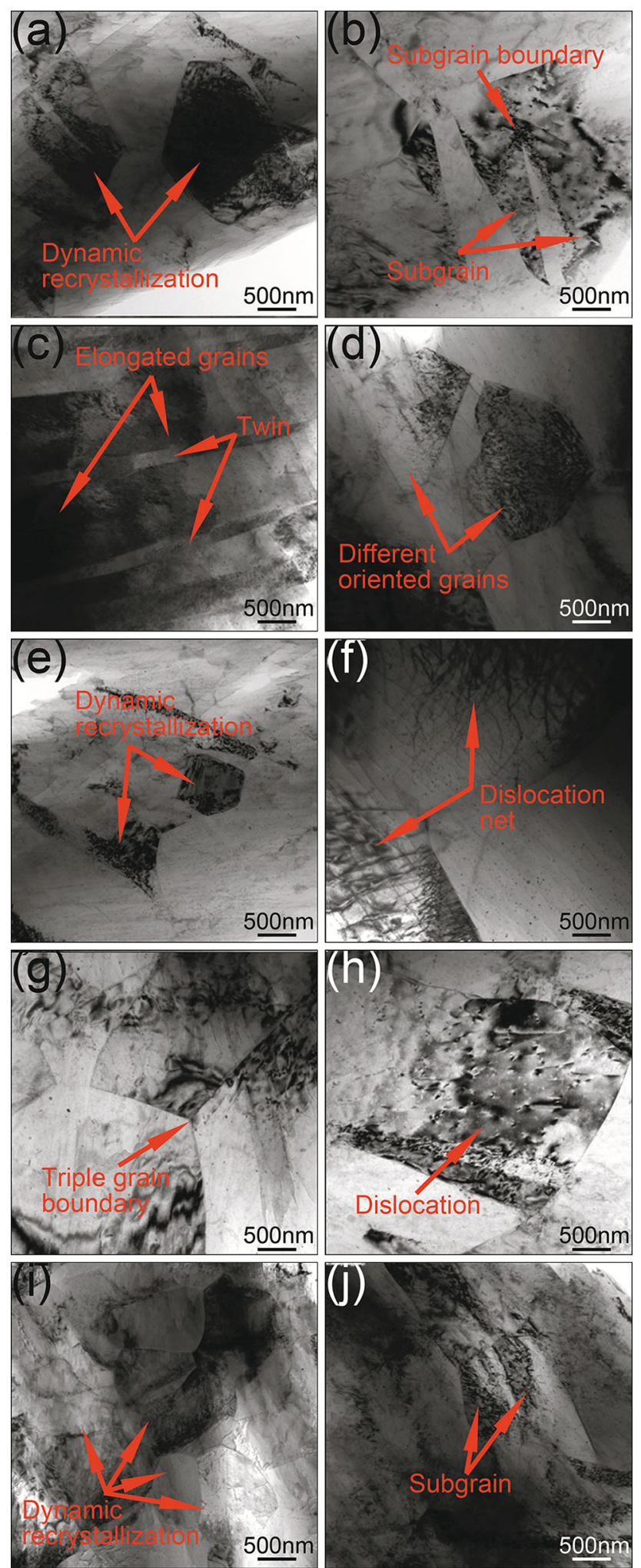

Fig. 8 TEM image of the characteristic locations: a DRX of location A, b subgrain of location A, c elongated grains and twin of location B, d different oriented grains of location $\mathrm{B}$, e DRX of location C, f dislocation net of location $\mathrm{C}, \mathbf{g}$ triple grain boundary of location $\mathrm{D}$, $\mathbf{h}$ dislocation of location D, $\mathbf{i}$ DRX of location E, $\mathbf{j}$ subgrain of location $\mathrm{E}$

\section{Conclusions}

The current work evaluated five characteristic locations microstructure evolution law of interrupted extrudate during AFE at $573 \mathrm{~K}$. The investigation educed the following conclusions.

(1) Compared with CE, the additional shear deformation could be provided by AFE owing to the special loading way. In addition to the die orifice, the shear deformation of the axis near the end of the punch was larger. With increasing punch down stroke, the shear deformation of each location along the axis was obviously higher than CE.

(2) Due to the double-split punches alternately applied load downward, the metal flow in each location in the billet was more complicated than CE. The material flow near the end of the fixed punch certainly produced vortex flow. The variation law of vortex size was consistent with the change of the range of the corner region between the double-split punches in the AFE.

(3) The grains at location $\mathrm{C}$ and location $\mathrm{E}$ along the axis were smaller and more homogeneous than location $\mathrm{D}$. The average grain size of location $\mathrm{E}$ was $4.73 \mu \mathrm{m}$, showing remarkable refined effect. At the same time, the average misorientation angle and the proportion of DRX were the largest.

(4) The dislocation density at the location D was lowest; however, the dislocations at the location $\mathrm{C}$ and location $\mathrm{E}$ were more serious from the observation of the grain morphology. This was consistent with the change of shear deformation in the corresponding location. Due to the complexity of the stress state and the flow behavior near the container wall, the microstructure observation remained to be further studied.

Acknowledgements This work was financially supported by the National Natural Science Foundation of China (No. 51675143).

\section{References}

[1] Q.S. Yang, Z.J. Yu, H.C. Pan, Q.W. Dai, J.H. Li, Acta Metall. Sin. (Engl. Lett.) 29, 475 (2016)

[2] F. Li, N. Bian, Y.C. Xu, X. Zeng, Mode. Phys. Lett. B 30, 1550261 (2016)

[3] Y. Yan, W.P. Deng, Z.F. Gao, J. Zhu, Z.J. Wang, X.W. Li, Acta Metall. Sin. (Engl. Lett.) 29, 163 (2016)

[4] K. Su, K.K. Deng, F.J. Xu, K.B. Nie, L. Zhang, X. Zhang, W.J. Li, Acta Metall. Sin. 28, 1015 (2015)

[5] B. Kim, H.P. Chan, H.S. Kim, B.S. You, S.S. Park, Scr. Mater. 76, 21 (2014)

[6] M. Chandrasekaran, Y. John, Mater. Sci. Eng. A 381, 308 (2004) 
[7] M. Shanzad, L. Wagner, Mater. Sci. Eng. A 506, 141 (2009)

[8] H. Borkar, M. Hoseini, M. Pekguleryuz, Mater. Sci. Eng. A 549, 168 (2012)

[9] T. Murai, S. Matsuoka, S. Miyamoto, Y. Oki, J. Mater. Process. Technol. 141, 207 (2003)

[10] H. Borkar, R. Gauvin, M. Pekguleryuz, J. Alloys Compd. 555, 219 (2013)

[11] F. Li, N. Bian, Y.C. XU, Mech. Res. Commun. 61, 27 (2014)

[12] F. Li, X. Zeng, G.J. Cao, Mater. Sci. Eng. A 639, 395 (2015)

[13] Q.S. Yang, B. Jiang, J.J. He, B. Song, W.J. Liu, H.W. Dong, F.S. Pan, Mater. Sci. Eng. A 612, 187 (2014)

[14] Y.J. Chen, Q.D. Wang, H.J. Roven, M.P. Liu, M. Karlsen, Y.D. Yu, J. Hjelen, Scr. Mater. 58, 311 (2008)

[15] Q.S. Yang, B. Jiang, Z.J. Yu, Q.W. Dai, S.Q. Luo, Acta Metall. Sin. (Engl. Lett.) 28, 1257 (2015)
[16] J.B. Lin, X.Y. Wang, W.J. Ren, X.X. Yang, Q.D. Wang, J. Mater. Sci. Technol. 32, 783 (2016)

[17] S.M. Fatemi-Varzaneh, A. Zarei-Hanzaki, Mater. Sci. Eng. A 504, 104 (2009)

[18] N. Haghdadi, A. Zarei-Hanzaki, D. Abou-Ras, M.H. Maghsoudi, A. Ghorbani, M. Kawasaki, Mater. Sci. Eng. A 595, 179 (2014)

[19] Z.Y. Zhang, H.S. Yu, S.Q. Wang, H. Yu, G.H. Min, J. Mater. Sci. Technol. 27, 159 (2011)

[20] Y.J. Chen, Q.D. Wang, J.G. Peng, C.Q. Zhai, W.J. Ding, J. Mater. Process. Technol. 182, 281 (2007)

[21] C.J. Wang, K.K. Deng, S.S. Zhou, W. Liang, Acta Metall. Sin. (Engl. Lett.) 29, 527 (2016)

[22] T. Al-Samman, X. Li, S.G. Chowdhury, Mater. Sci. Eng. A 527, 3450 (2010) 